\title{
Selection of ERP in Deterministic Unpredictable Scenario
}

\author{
G. R. Gosavi ${ }^{1}$, Dr. V. M. Thakare ${ }^{2}$ \\ Department of Computer Engineering, Dr. Panjabrao Deshmukh Polytechnic, Amravati, Maharashtra, India ${ }^{1}$ \\ Department of Computer Science \& Engineering, Sant Gadge Baba Amravati University, Maharashtra, India ${ }^{2}$
}

\begin{abstract}
ERP, The Enterprise resource planning is a planning philosophy, enabled with software that attempts to integrate all the business processes of various departments and functions across a Company on to a Software system that can serve the needs to execute the transactions of the tightly integrated departments. Determinism is the incidental concept that states that every happening and choice is a consequence of and is eventually determined by archived but live causes and reasons. Determinism is often taken to incorporate liberty of selection because it embodies the principal of past actions and future results. In mathematics all deterministic processes are predictable, you get to understand the next state, given the current state, and then if owned a strong computing resource, one ought to produce future step derivations. One example is count on the constraints laying precisely for an ERP today and has prediction of ERP selection suiting the constraints in unpredictable scenario. For an archived set of an ERP, or numerous ERPs, a set of observations on a variable measured at successive points of time are recorded for performance and implementation progress, then for every future procurement and deployment of that ERP, the recorded trend may guide the future decisions. The polynomial representation of coded ERP constraints based matrix can be simplified and solved using least squares method of regular moving averages. The trends in history can predict the trends and success of ERP in present or future in mathematically modeled processing form. This paper analyses the simulation logic with a practical case study, in deterministic unpredictable scenario.
\end{abstract}

Keywords: MMS, POMS, Inventory Management System (IMS), Human Resource Development Management System (HRDMS), Payroll Management System (PMS), Electrical Management System (EMS)

\section{INTRODUCTION}

ERP, The Enterprise resource planning is a planning philosophy, enabled with software that attempts to integrate all the business processes of various departments and functions across a Company on to a Software system that can serve the needs to execute the transactions of the tightly integrated departments, Precisely, The Enterprise resource planning software is a consolidated group of individual software, that serve and automate the actual or manual enterprise management systems that are comprised within an ideal organization, the organization could be a manufacturing unit or design consultancy or even a service rendering industry.

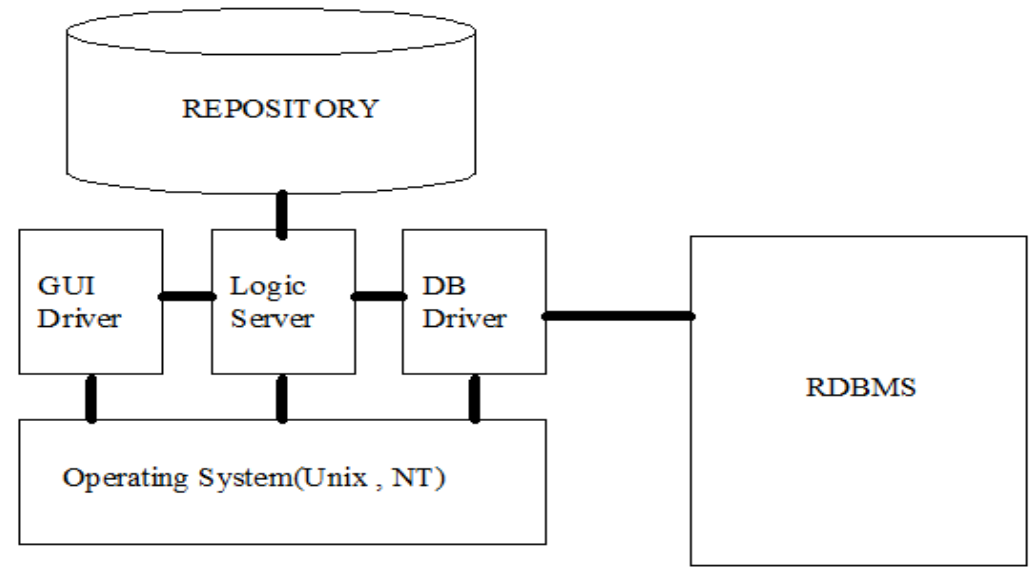

Figure 1 ERP Structure

An ERP generally encompasses, ready to implement core software's for automating the shop floor systems, like, Marketing Management System (MMS), Purchase Order Management System (POMS), Inventory Management System (IMS), Accounts Management System (AMS), Human Resource Development Management System (HRDMS), Payroll 
Management System (PMS), Mechanical Maintenance Management System (MMMS), Electrical Management System (EMS), Dispatch Management System (DMS), Quality Control Management System (QCMS), etc. i.e. in Logistics, Maintenance, Sales and Distribution, Quality management, Materials management, Human Resources, Manufacturing and in Strategic and Operational planning. ERP covers the techniques and the concepts employed for the integrated management of the business as a whole, with the viewpoint of automating the TPS (Transaction Processing Systems), by applying the concepts of software engineering and Business Engineering together, to improve the efficiency of an enterprise.

ERP, as shown above, is a multitier architecture with central data repository, drivers and a database system mounted on an operating system. Below shown is the process framework of an ERP. The Business strategy form business model which in turn conceives the relevant enterprise management system processes. The database and interaction processes make an ERP a complete entity.

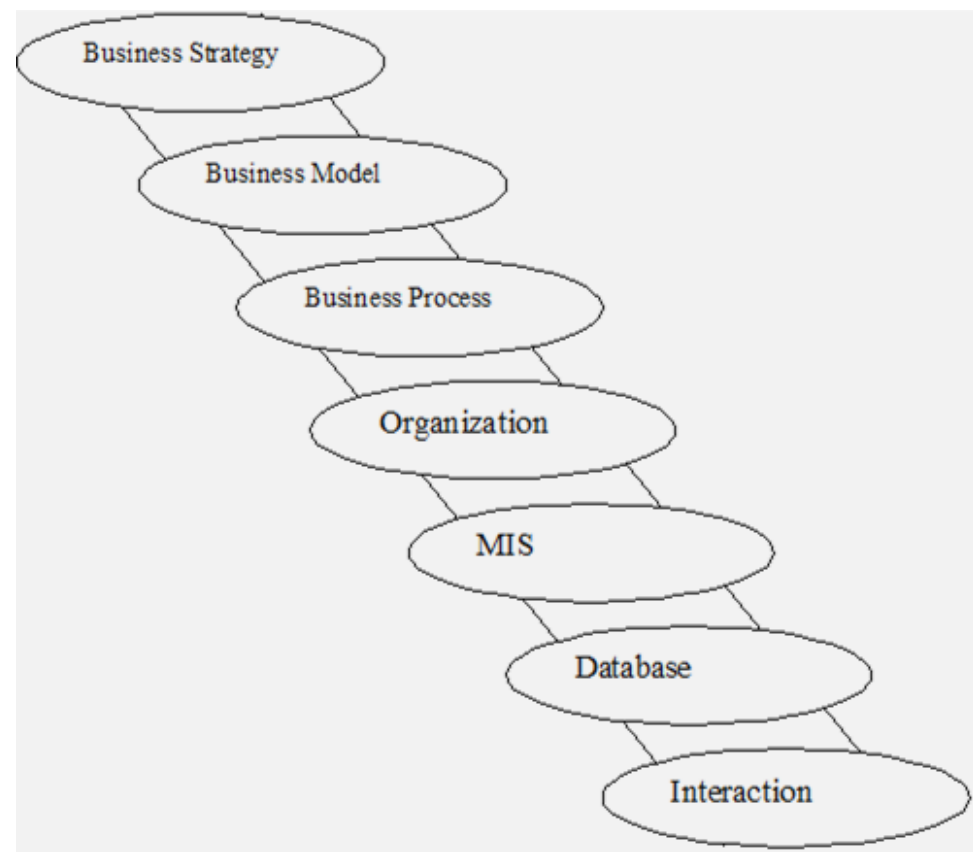

Figure 2 ERP, a Process framework.

Determinism is the incidental concept that every happening and choice is a consequence of and is eventually determined by archived but live causes and reasons. Determinism is often taken to incorporate liberty of selection because it embodies the principal of past actions and future results. Rationally the theory proceeds with the principal that knowledge, wisdom and analysis may lead to coping results. In case of an ERP, deterministic unpredictability of choice of ERP package, implementation methodology and yield generation can invariably be a function of comprehensive set of input parameters. Deterministic framework over the years of thought platforms have sprung from versatile and sometimes matching purposes and viewpoints. Determinism frequently refers to causal determinism, which in science is known as cause-and-effect. In ERP, there are ample of attributes to decide on the ERP deployment in an organization, own constraints too. It is the concept that events within a given organizational ERP paradigm, and are vault by causality in such a way that any state of an ERP object or event in the form of Organizational fore view is completely determined by prior comprehensive thought. Other debates often concern the scope of determined systems, but here in purview of an ERP, which can be treated as a single determinate system may lead to debate concerning determinism as compatibility or in compatibility as Determinism often requires that perfect prediction be practically possible. ERP choice and deployment has invariably been tried to be accommodated within the norms of deterministic unpredictable scenario. The selection of an ERP in an organization often implies unpredicted deterministic strategic methodology.

\section{ERP SELECTION - A DETERMINISTIC APPROACH}

The intense reasons behind using mathematical interpretation for deterministic unpredictability in principal applied to ERP taking into actions are, in mathematics all deterministic processes are predictable, you get to understand the next state, given the current state, and then if owned a strong computing resource, one ought to produce future step derivations. One example is count on the constraints laying precisely for an ERP today and has prediction of ERP 
selection suiting the constraints in unpredictable scenario. Thus, provided with an input model, one can manipulate to, in principle, the time evolution of such a model to any future existential state. This can lead to increasingly complex results that can be controlled with preclusion of parity and precision. The uncertainty associations are pact with inspection measures of conjugate factors and parameters. Measuring one variable sets restrictions on the measurement of the other variable. One can measure the impact of the selection parameter to any desired precision, and, simultaneously gather more and more information about available ERPs with features information subsets. Predictability is here connected to the interpretation of the best fit ERP model and its implementation strategy, based on input sets of constraints. In such uncertainty vulnerability, without mathematical modeling, there is not a better "prediction" possible. If there is some missing information, the selection prediction may have precision margin.

\section{SET OF CONSTRAINT EXPRESSIONS}

For an archived set of an ERP, or numerous ERPs, a set of observations on a variable measured at successive points of time are recorded for performance and implementation progress, then for every future procurement and deployment of that ERP, the recorded trend may guide the future decisions, howsoever unpredictable the scenario may be. Quantitative data collected from field may be used in specific forms to play vital role in future decision making. A variable $t$ represents time over which the parametric trend y can be recorded. The peculiarities to be recorded over time for an ERP could be categorized in four important components.

- Regular Secular or long term trend

- recurrent fuzzy variation

- Cyclical iterative fluctuation

- lopsided zigzag or random faction

In the conventional classical approach, it is precluded from the established mathematical theory, that the noted value $\mathrm{y}$, may be represented either as the product of the above gradients i.e.

$\mathrm{y}_{\mathrm{i}}=\mathrm{T} \times \mathrm{S} \times \mathrm{C}$ X I (multiplicative model)

or as the addition of components

$\mathrm{y}_{\mathrm{i}}=\mathrm{T}+\mathrm{S}+\mathrm{C}+\mathrm{I}$ (additive model)

Both models are used based on severity of results solicited. Conventional Trend is an apparent stable and regular permanent change in state of an ERP, its subtle databases, designed interactive screens and processing logic. The code, functions, argument passing, variables, constants, data types, data structures and to some extent logic has the given state of change, recorded either as a variable or a polynomial. The indicative representation in the form of a variable or polynomial actually points to a snapshots state or database state at given point of time, especially when a trend is noted. Recurrent Fuzzy trend recording refers to the periodic elicitation of all types of architectural components of an ERP, e.g. firmware, connectivity logic, data propagation terms etc. where the period of recording repeats at regular intervals or periods of time. Cyclic Fluctuation includes those factors leading to alternating periods of expansion and contraction that characterize major updating in an ERP. The important components of ERP here are backups, mirroring and replications of databases.

Lopsided Zigzag methodology includes all components, all factors not classifiable elsewhere. Thus, factors such as modifications, customizations, third party software add ons and patch ups, evaluation version software associated are covered for trends noting in an ERP.

Methods of Measurement of trends in the most scientific ways proposed are, Regular Moving Average Method, Method of compatibility by Fitting Polynomials. In Regular moving average method, an apparently regular trend in parameters of an ERP for a given implementation is proposed to be obtained by smoothing out fluctuations by the process of averaging. In the latter, a polynomial of suitable degree is elected either for the original variables or for its transformed variables, where variables refer to objects or real time embodiment of ERP snapshots and its derivatives are determined by the method of least squares. The choice of the degree of the polynomial can be made by plotting the data on a graph paper where different scales, arithmetic, semi-logarithmic or double-logarithmic scales may be used. Measurement of trend is necessary for noting state of progress of an ERP over time and for forecasting the future. The real challenge here to accumulate ERP variables set into a compact polynomial set. Prima facie, the objective is to preserve genuine information in trend. 
Regular Moving Average Method is a less complicated method of smoothing out fluctuations of a series by calculating a number of averages to fan the multiple periods of the noting time span. The first step consists in selecting proper frequency of the regular moving average. If the period chosen is $n$ units, the moving averages are obtained by calculating a series of mean values of $n$ successive values casing overlapping periods of the ERP snapshots chain. Denoting the original chain by $\mathrm{y}_{1}, \mathrm{y}_{2}, \mathrm{y}_{3} \ldots ., \mathrm{y}_{\mathrm{n}}$ the mean of the first $\mathrm{n}$ values, given by

Snapshot $1=\mathrm{y}_{1}+\mathrm{y}_{2}+\mathrm{y}_{3}+\mathrm{y}_{\mathrm{n}}$

Snapshopt $1=\sum_{\mathrm{i}=1}^{\mathrm{L}} \mathrm{yi} / \mathrm{i}$

Regular Moving average method is straightforward to apply but the accomplishment of this method depends on the proper choice of algorithm used for sampling the snapshot. Regular Moving average with a macro recording period exactly equal to or a multiple of the period of the cycle present in the prospective change series of an ERP will completely or closely eliminate the random component and give an estimate of the trend. This method is quite efficient and moldable but some trend values at the beginning and at the end of the ERP implementation cycle have to be left out and their number increases with increase in the period of the adhoc start stoppage in implementation cycle of an ERP. e.g. Some big organizations strategically stop implementation periodically thus disturbing the averages on snapshots sampling.

Method of fitting polynomials is perhaps the best and the most objective method of determining ERP trend. Here is an appropriate type of polynomial is selected for trend and the constants appearing in the trend equation are determined on the basis of the state of design, development, implementation or procession of an ERP. The choice of an appropriate polynomial is facilitated by a guided grid of representation of the ERP data for which, apart from the usual arithmetic scales, semi logarithmic or doubly logarithmic scales may be used. If the consolidated data show approximately geometric figure bent on plotting, the equations used are appropriate geometric equation for that particular trend, which may be derived by taking logarithm of

$$
\mathrm{Y}=\mathrm{A} \cdot \mathrm{B}^{\mathrm{x}} \quad \text { represented in the exponential function form. }
$$

where $\mathrm{a}=\log \mathrm{A}$ and $\mathrm{b}=\log \mathrm{B}$.

Some times a second or a third degree polynomial may also be fitted. $\mathrm{Y}=\mathrm{a}+\mathrm{bx}+\mathrm{cx}^{2}$

Representing second degree polynomial or parabola geometric figure $\quad \mathrm{Y}=\mathrm{a}+\mathrm{bx}+\mathrm{cx}^{2}+\mathrm{dx}^{3}$

Represent third degree polynomial, The constants appearing in the above equations such as a, b, c, .... are obtained by applying the principles of "least squares", using regression. It is evident that the values of the constants formed will be such as to make the sum of squares of the deviations.

$\mathrm{Z}(\mathrm{y}-\mathrm{Y})^{2}$ minimum

where, $y=$ observed value

$\mathrm{Y}=$ anticipated value obtained from the trend equation of the type represented by state of ERP Which may be of type

$\mathrm{Y}=\mathrm{a}+\mathrm{bx}$ or

$\mathrm{Y}=\mathrm{a}+\mathrm{bx}+\mathrm{cx^{2 }}$

and the summation is taken over all the observations. In the case of a straight line fitted by the method of least squares, the constants $\mathrm{a}$ and $\mathrm{b}$ are resolute by the following normal equations:

$\sum \mathrm{y}=\mathrm{na}+\mathrm{b} \sum \mathrm{x}$

$\sum x y=a \sum x+b \sum x^{2}$

Where $\mathrm{n}$ is the number of units of time of taking sampling snapshots of an ERP.

Likewise, in the case second degree polynomial the constants $\mathrm{a}, \mathrm{b}$ and $\mathrm{c}$ are determined from the three normal equations

$\sum \mathrm{y}=\mathrm{na}+\mathrm{b} \sum \mathrm{x}+\mathrm{c} \sum \mathrm{x}^{2}$

$\sum x y=a \sum x+b \sum x^{2}+c \sum x^{3}$

$\sum x^{2} y=a \sum x^{2}+b \sum x^{3}+c \sum x^{4}$ 
Vol. 8, Issue 5, May 2019

As per the rules of established mathematical principles, and the rules for writing down the normal equations, to get the commencing normal equation, multiply each surveillance by coefficient of a in that equation then sum up over all the $n$ observations for that ERP sampling.

Thus the first normal equation can be represented in the form

$$
\sum \mathrm{y}=\mathrm{na}+\mathrm{b} \sum \mathrm{x}
$$

To get the second normal equation, multiply each observation by the coefficient of $b$ in that equation and sum up all the $\mathrm{n}$ observations. e.g. coefficient of $\mathrm{b}$ is $\mathrm{x}$.

$\sum x y=a \sum x+b \sum x^{2}$

\section{A CASE INVESTIGATION}

In mathematics, a matrix polynomial is a polynomial with square matrices as variables. Therefore a scalar valued polynomial is represented as below.

$p(x)=\sum_{i=1}^{n} a_{i} x^{i}=a_{0}+a_{1} x+a_{2} x^{2}+\ldots . .+a_{n} x^{n}$

The matrix evaluation interpretation is

$P(A)=\sum_{i=1}^{n} a_{i} A^{i}=a_{0} I+a_{1} A+a_{2} A^{2}+\ldots+a_{n} A^{n}$

where I is identity matrix

The representation of an ERP state in the polynomial form $\mathrm{P}=\mathrm{ax} \mathrm{x}^{2}+\mathrm{bx}+\mathrm{c}$ is a derivation of matrix form

$$
\begin{aligned}
\mathrm{P}=\mathrm{ax}^{2}+\mathrm{bx}+\mathrm{c}= & \left(\begin{array}{l}
\mathrm{Ax}^{2} \\
\mathrm{bx} \\
\mathrm{c}
\end{array}\right) \\
& =\left(\begin{array}{ccc}
\mathrm{x} & \mathrm{x} & \mathrm{a} \\
1 & \mathrm{x} & \mathrm{b} \\
1 & 1 & \mathrm{c}
\end{array}\right) X\left(\begin{array}{l}
1 \\
1 \\
1
\end{array}\right)
\end{aligned}
$$

If the nine cells of matrix represent 9 trends of a single or more parameters of ERP that act as trend or decision maker, then, e.g. trend of implementation time of Inventory and Purchase order management module of an ERP Lets assume trend values as

\begin{tabular}{|l|l|l|l|l|l|l|l|l|l|}
\hline Trend & 1 & 2 & 3 & 4 & 5 & 6 & 7 & 8 & 9 \\
\hline Value & 18 & 21 & 24 & 32 & 27 & 39 & 44 & 49 & 78 \\
\hline
\end{tabular}

Where trend represents the trait on time line while value represents state of ERP in coded form. A separate code sheet can be maintained for coded values. The coding is not within the purview of this paper to reduce the volume. In order to predict tenth trend state of the trend 'Implementation time of Inventory and Purchase order management module of an ERP ‘ for current ERP, Fitting straight line trend will be

\begin{tabular}{|c|c|c|c|c|}
\hline Trend & Value(y) & $\mathbf{X}$ & $\mathbf{X}^{\mathbf{2}}$ & $\mathbf{x y}$ \\
\hline $\mathbf{( 1 )}$ & $\mathbf{( 2 )}$ & $\mathbf{( 3 )}$ & $\mathbf{( 4 )}$ & $\mathbf{( 5 )}$ \\
\hline 1 & 18 & -4 & 16 & -72 \\
\hline 2 & 21 & -3 & 9 & -63 \\
\hline 3 & 24 & -2 & 4 & -48 \\
\hline 4 & 32 & -1 & 1 & -32 \\
\hline 5 & 27 & 0 & 0 & 0 \\
\hline 6 & 39 & 1 & 1 & 39 \\
\hline 7 & 44 & 2 & 4 & 88 \\
\hline 8 & 49 & 3 & 9 & 147 \\
\hline 9 & 78 & 4 & 16 & 312 \\
\hline Total & $\mathbf{3 3 2}$ & $\mathbf{0}$ & $\mathbf{6 0}$ & $\mathbf{3 7 1}$ \\
\hline
\end{tabular}




\section{RESULTS AND DISCUSSION}

Here calculation of a and $\mathrm{b}$ constants can be done as

$9 \mathrm{a}=332$ therefore value of $\mathrm{a}=36.88$

$60 \mathrm{~b}=371$ therefore value of $b=6.18$

The method of fitting polynomials completely eliminates personal bias and trend values for all the given periods can be obtained. With least squares method, the trend equation, the estimate for tenth trend, i. e. 'Implementation time of Inventory and Purchase order management module of an ERP' is decided as follows

The increment of trend $\mathrm{x}$ is $=1$

And the value of $x$ for tenth trend will be 5 ,

Thus, The trend equation is $\mathrm{Y}=36.88+6.18 \mathrm{x}$

$\mathrm{Y}=36.88+(5 \mathrm{X} 6.18)$

$\mathrm{Y}=67.78$

which can be rounded to tally the deterministically predicted trend in code sheet for the ERP on predecided constraint, in unpredictable scenario.

\section{CONCLUSION}

ERP, as shown above, is a multitier architecture with central data repository, drivers and a database system mounted on an operating system. . In ERP, there are ample of attributes to decide on the ERP deployment in an organization. ERP choice and deployment has invariably be tried to be accommodated within the norms of deterministic unpredictable scenario, when, the selection of an ERP in an organization often implied unpredicted deterministic strategic methodology. Provided with an input model, one can manipulate to, in principle, the time evolution of such a model to any future existential state. . Predictability is here connected to the interpretation of the best fit ERP model and its implementation strategy, based on input sets of constraints. In such uncertainty vulnerability, without mathematical modeling, there is not a better "prediction" possible. For an archived set of an ERP, or numerous ERPs, a set of observations on a variable measured at successive points of time are recorded for performance and implementation progress, then for every future procurement and deployment of that ERP, the recorded trend may guide the future decisions, howsoever unpredictable the scenario may be. Four peculiar polynomial components are usable. . If the period chosen is $n$ units, the moving averages are obtained by calculating a series of mean values of $n$ successive values casing overlapping periods of the ERP snapshots chain. Regular Moving average method is straightforward to apply but the accomplishment of this method depends on the proper choice of algorithm used for sampling the snapshot. Appropriate type of polynomial is selected for trend and the constants appearing in the trend equation are determined on the basis of the state of design, development, implementation or procession of an ERP. The choice of an appropriate polynomial is facilitated by a guided grid of representation of the ERP data for which, apart from the usual arithmetic scales, semi logarithmic or doubly logarithmic scales are used. Matrix representation of historical trend is expressed in the form of a polynomial, which in turn made subject to least squares method, the trend equation and the estimate for present or future trend is decided.

\section{REFERENCES}

[1] Alexis Leon "Enterprise Resource Planning ",Tata Mc Graw Hill Publishing Company Ltd. Twelfth Reprint 2004 , pp 27 - 98

[2] Vinodkumar Garg, N. K. Venkitakrishnan “Enterprise Resource Planning, Concepts and Planning”,,II Edition, PHI 2004, pp 112 - 147

[3] Golub, G.H., \& C.F. Van Loan, “An analysis of the total least squares problem”, SIAM Jour on Numerical Analysis 17 (6), pp. 883-893, 1980.

[4] Stefan Rummens, Stefaan E.Cuypers, "Determinism \& the Paradox of Predictability" Springer Erkenntnis Mar2010, Vol72, Iss2, pp233-249

[5] Dario Bini \& Victor Pan, "Polynomial \& Matrix Computations: Fundamental Algm”, Springer Science + Business media LLC 1994 , pp 47-76.

[6] E j barbeau, "Polynomials", Springer book isbn 0-38740627-1, pp 102-111

[7] Rahul V. Altekar "Enterprise Resource Planning, Theory and Practice", PHI 2004, pp100-129

[8] C. Heij, J. C. Willems, “A Deterministic Approach to Approximate Modelling”, springer link books, pp 49-134

[9] Heij, C., "Approximate modelling of deterministic systems, in Curtain - Modelling, Robustness and Sensitivity Reduction in Control Systems", NATO ASI Series, Springer, Berlin, 1987, pp. 271-283

[10] Kalman, R.E., P.L. Falb and M.A. Arbib, Topics in Mathematical System Theory, McGraw-Hill, New York, 1969.

[11] Ljung, L., \& P.E. Caines, “Asymptotic normality of prediction error estimators for approximate system models, Stochastics “ pp. $29-46,1979$.

[12] Bishop, R. C.,"On separating predictability and determinism”, Springer Erkenntnis, 58, 169-188.

[13] Stout Edgar Lee Stout, "Polynomial Convexity", Publisher: Birkhauser Boston,ISBN: 9780817645373, 0817645373 , Edition: $1^{\text {st }}$., pp 134 - 177

[14] Dr. Subodh Kesharwani "ERP Systems, Experiences, and Upsurges ”, Pragati Prakashan, First Edition 2004, pp 78 - 111

[15] Karl Peterson "The Optimization Techniques", HRMS and Group, 1995, pp 123-166 